\title{
Autonomic nervous system dysfunction in narcolepsy type 1: time to move forward to the next level?
}

\author{
Alessandro Silvani ${ }^{1}$ \\ Received: 29 October 2020 / Revised: 30 October 2020 / Published online: 12 November 2020 \\ (c) Springer-Verlag GmbH Germany, part of Springer Nature 2020
}

Keywords Autonomic $\cdot$ Sleep $\cdot$ Narcolepsy $\cdot$ Sudoscan $\cdot$ Electrochemical skin conductance

Narcolepsy, although a rare disease, is one of the most common causes of chronic sleepiness. Patients with narcolepsy type 1 (NT1) typically show cataplexy and a dramatic reduction in the cerebrospinal fluid concentration of the neuropeptide orexin A (hypocretin 1), which results from extensive loss of the orexin-producing neurons in the hypothalamus [1].

The orexin neurons are a critical component of the sleep control circuits and an integral part of the central autonomic network [1, 2]. In 1982, Sachs and Kaiser reported smaller increases in heart rate (HR) and blood pressure (BP) at the handgrip test and smaller changes in HR during deep breathing and the Valsalva maneuver, but no significant alteration at the head-up tilt test (HUTT) and the cold face test [3], in patients with NT1 compared with healthy controls. However, their findings were not confirmed in later studies by different groups $[4,5]$. On the other hand, higher scores of a questionnaire on autonomic symptoms (the Scales for Outcomes in Parkinson's Disease-Autonomic Dysfunction, SCOPA-AUT) have been recently reported in patients with NT1 than in healthy controls, with significant impairments in the gastrointestinal, urinary, cardiovascular, thermoregulatory, pupillomotor, and sexual subdomains [6].

In this issue of Clinical Autonomic Research, Rocchi and colleagues improved over previous work by administering a comprehensive set of autonomic cardiovascular function tests, an electrochemical skin conductance (ESC) test of sudomotor function, and the SCOPA-AUT questionnaire to a sample of patients with NT1 and controls [7]. They found that, compared with controls, patients with NT1

Alessandro Silvani

alessandro.silvani3@unibo.it

1 Department of Biomedical and Neuromotor Sciences, University of Bologna, Piazza di Porta San Donato 2, 40126 Bologna, Italy showed significantly higher systolic BP during supine rest and HUTT, significant HR alterations during HUTT, the cold-face test, the Valsalva maneuver, and deep breathing, significantly lower ESC in the hands, and higher scores for all SCOPA-AUT subdomains. Based on these results, Rocchi and colleagues concluded for the occurrence of blunted parasympathetic activity during wakefulness, mild sudomotor dysfunction, and a large variety of autonomic symptoms in patients with NT1 [7].

The study by Rocchi and colleagues has limitations, acknowledged by the authors [7]. Patients who smoked or had an apnea-hypopnea index greater than five or assumed drugs to treat NT1 were excluded from the study [7]. This was both a point of strength and a limitation, as it decreased within-group variability at the expense of the generalizability of the findings, with a potential for underestimation of autonomic dysfunction of patients with NT1. Paradoxically, however, systolic BP during HUTT and SCOPA-AUT scores were much higher in the patients with NT1 studied by Rocchi et al. $(175.1 \pm 28.9 \mathrm{mmHg}$ and $20.6 \pm 8.4$, respectively [7]; mean \pm SD) than in patients with NT1 included in previous reports $(128 \pm 20 \mathrm{mmHg}$ [5] and 12.92 \pm 6.84 [6], respectively). Moreover, the patients with NT1 and the controls in the study by Rocchi and colleagues were not matched for body mass index (BMI) [7]. The non-significant difference in BMI between groups $(28.4 \pm 7.6$ vs. $24 \pm 3.8$ in patents with NT1 vs controls, $P=0.1$ [7]), should be interpreted considering the low sample size (12 patients with NT1 and 14 controls), the lack of a statistical power analysis, and the known association between NT1 and obesity [1]. These considerations on BMI may be relevant to the estimation of autonomic dysfunction, as Rocchi and colleagues reported a positive correlation between BMI and systolic BP during HUTT [7]. Finally, the occurrence, reported by Rocchi and colleagues, of a sudomotor function impairment in the upper but not in the lower extremities of patients with NT1 [7] is 
unusual, and the validity of sudomotor function assessment based on ESC is debated [8].

These limitations notwithstanding, the study by Rocchi and colleagues [7] has the merit of bringing to the fore again the links between NT1 and autonomic dysfunction, including, but not limited to, the cardiovascular autonomic domain. It may now be time to take stock and move forward to the next level. Did discrepancies among published reports [3-5, 7] reflect type II statistical errors in the studies with negative results $[4,5]$, which concealed a true general association between NT1 and autonomic cardiovascular dysfunction? One of the latter studies did detect alterations in HR variability in patients with NT1 during HUTT [5]. Alternatively, autonomic cardiovascular dysfunction may occur only in some patients with NT1, which could have been preferentially sampled by chance in the small-size studies by Sachs and Kaiser [3] and Rocchi and colleagues [7]. Even if this were the case, the conclusion would be quite relevant nevertheless, for it may be linked to the increased risk of stroke, myocardial infarction, coronary revascularization, cardiac arrest, and heart failure, which has been recently reported for patients with narcolepsy [9]. Given the rarity of narcolepsy and the substantial skill and resources required for objective autonomic testing, international multicenter studies would probably be needed to achieve sufficient statistical power and to account efficiently for the effects of age, sex, BMI, smoking, and comorbidities including obstructive sleep apnea, which is prevalent in adult patients with NT1 [10]. Obstructive sleep apnea exacerbates cardiovascular alterations during nighttime sleep, which also characterize NT1 and may further contribute to cardiovascular risk [11]. Medications to treat NT1 may also be associated with significant cardiovascular effects $[12,13]$. Recruitment of both untreated and treated patients with NT1 would increase the generalizability of the results on the autonomic function to patients with NT1 in real-life conditions, and, together with assessment of residual orexin levels in the cerebrospinal fluid, would help disentangle potential autonomic and cardiovascular consequences of daytime sleepiness from those of orexin deficiency.

Funding None.

\section{References}

1. Grimaldi D, Silvani A, Benarroch EE, Cortelli P (2014) Orexin/ hypocretin system and autonomic control: new insights and clinical correlations. Neurology 82(3):271-278. https://doi. org/10.1212/WNL.0000000000000045

2. Bastianini S, Silvani A (2018) Clinical implications of basic research role of hypocretin/orexin neurons in the central autonomic network. Clin Trans Neurosci. https://doi. org/10.1177/2514183X18789327

3. Sachs C, Kaijser L (1982) Autonomic regulation of cardiopulmonary functions in sleep apnea syndrome and narcolepsy. Sleep 5(3):227-238. https://doi.org/10.1093/sleep/5.3.227

4. Hublin C, Matikainen E, Partinen M (1994) Autonomic nervous system function in narcolepsy. J Sleep Res 3:131-137. https://doi. org/10.1111/j.1365-2869.1994.tb00119.x

5. Grimaldi D, Pierangeli G, Barletta G, Terlizzi R, Plazzi G, Cevoli S, Franceschini C, Montagna P, Cortelli P (2010) Spectral analysis of heart rate variability reveals an enhanced sympathetic activity in narcolepsy with cataplexy. Clin Neurophysiol 121:1142-1147. https://doi.org/10.1016/j.clinph.2010.01.028

6. Barateau L, Chenini S, Evangelista E, Jaussent I, Lopez R, Dauvilliers Y (2019) Clinical autonomic dysfunction in narcolepsy type 1. Sleep 42(12):187. https://doi.org/10.1093/sleep/zsz187

7. Rocchi C, Placidi F, del Bianco C, Liguori C, Pisani A, Mercuri NB (2020) Izzi F (2020) Autonomic symptoms, cardiovascular and sudomotor evaluation in de novo type 1 narcolepsy. Clin Auton Res. https://doi.org/10.1007/s10286-020-00718-w

8. Vinik AI, Casellini CM, Parson HK (2019) Electrochemical skin conductance to measure sudomotor function: the importance of not misinterpreting the evidence. Clin Auton Res 29:13-15. https ://doi.org/10.1007/s10286-018-0562-7

9. Black J, Reaven NL, Funk SE, McGaughey K, Ohayon MM, Guilleminault C, Ruoff C (2017) Medical comorbidity in narcolepsy: findings from the burden of narcolepsy disease (BOND) study. Sleep Med 33:13-18. https://doi.org/10.1016/j.sleep.2016.04.004

10. Sansa G, Iranzo A, Santamaria J (2010) Obstructive sleep apnea in narcolepsy. Sleep Med 11(1):93-95. https://doi.org/10.1016/j. sleep.2009.02.009

11. Berteotti C, Silvani A (2017) The link between narcolepsy and autonomic cardiovascular dysfunction: a translational perspective. Clin Auton Res 28(6):545-555. https://doi.org/10.1007/s1028 6-017-0473-z

12. Bosco A, Lopez R, Barateau L, Chenini S, Pesenti C, Pépin J-L, Jaussent I, Dauvilliers Y (2018) Effect of psychostimulants on blood pressure profile and endothelial function in narcolepsy. Neurology 90(6):e479-e491. https://doi.org/10.1212/WNL.00000 00000004911

13. Bušek P, Bušková J, Miletínová E, Mladá K, Nevšímalová S, Šonka K (2018) Modafinil reduces parasympathetic activity but does not influence autonomic reactivity to orthostatic load in narcolepsy type 1. Clin Neuropharmacol 41(4):111-115. https://doi. org/10.1097/WNF.0000000000000287

\title{
Compliance with ethical standards
}

\author{
Conflict of interest None.
}

